Supporting Information

\title{
Atomistic Observation of Desodiation induced Phase Transition in Sodium Tungsten Bronze
}

Qi Meng, ${ }^{+}$Yuanlin Zhuang, ${ }^{+}$Renhui Jiang, ${ }^{+}$Shuang Meng, ${ }^{+}$Zhengzhou Wang, ${ }^{+}$Lei Li, ${ }^{+}$ Ying Zhang, ${ }^{\dagger}$ Shuangfeng Jia, ${ }^{\dagger}$ Peili Zhao, ${ }^{\dagger}$ He Zheng, $, \square,+, \neq$, J Jianbo Wang ${ }^{\square, t}$

tSchool of Physics and Technology, Center for Electron Microscopy, MOE Key Laboratory of Artificial Micro- and Nano-structures, and Institute for Advanced Studies, Wuhan University, Wuhan 430072, China

₹Suzhou Institute of Wuhan University, Suzhou, Jiangsu 215123, China

\$Wuhan University Shenzhen Research Institute, Shenzhen, Guangdong 518057, China

“Corresponding authors. Emails: wang@whu.edu.cn (J. W.); zhenghe@whu.edu.cn (H.

Z.) 

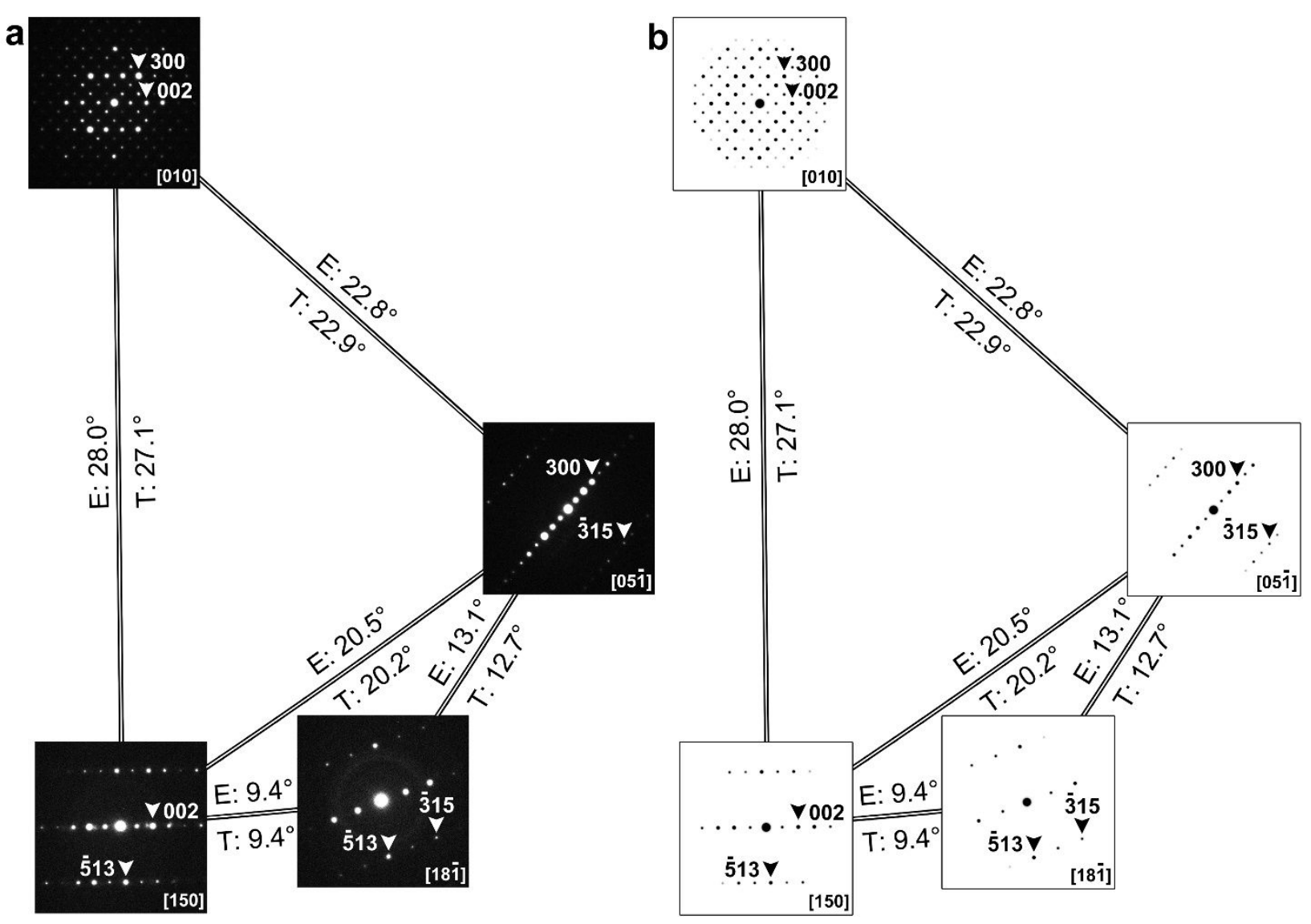

Figure S1. Serial experimental and corresponding simulated diffraction patterns of $\mathrm{Na}_{0.5} \mathrm{WO}_{3.25}$ 

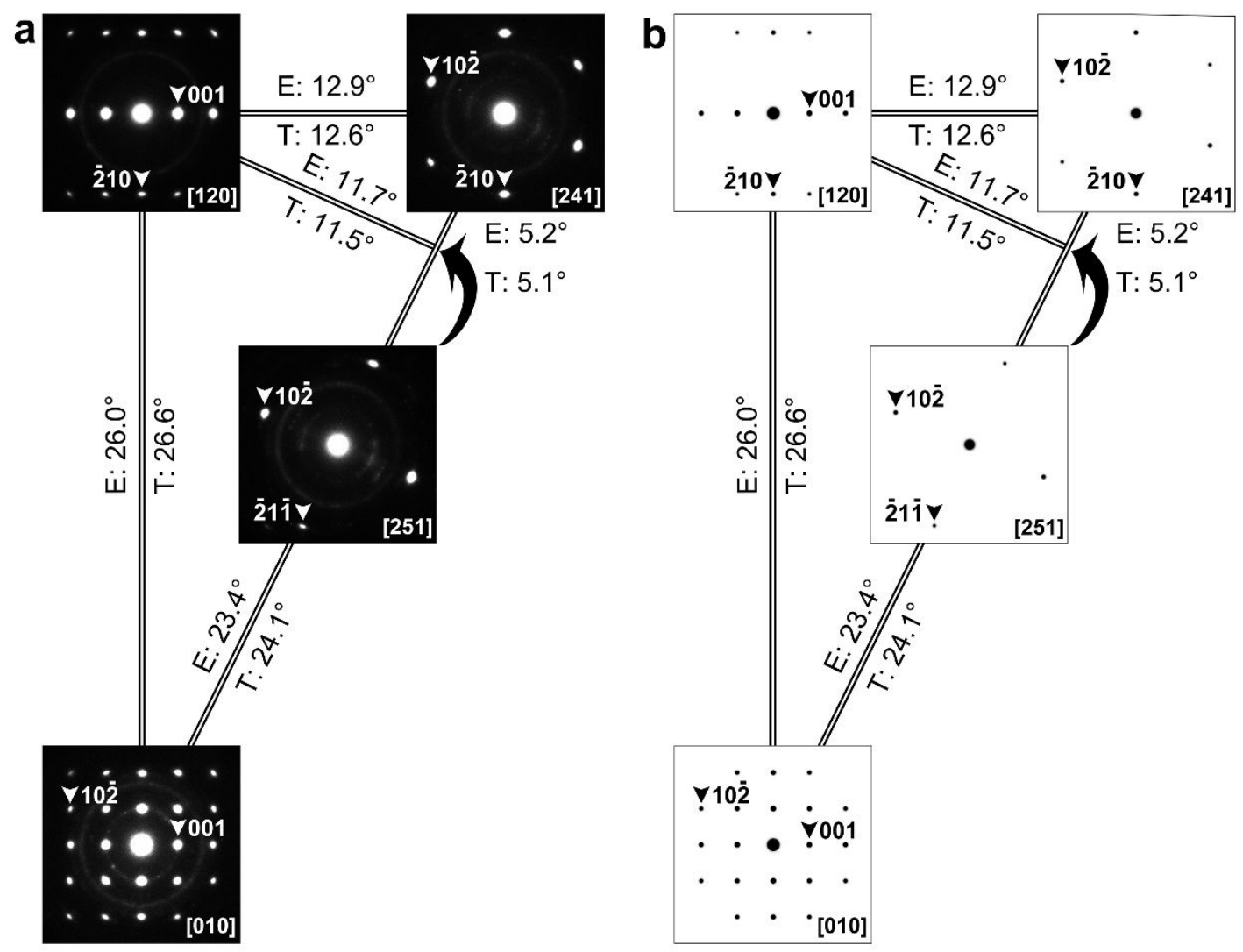

Figure S2. Serial experimental and corresponding simulated diffraction patterns of cubic $\mathrm{Na}_{x} \mathrm{WO}_{3}$
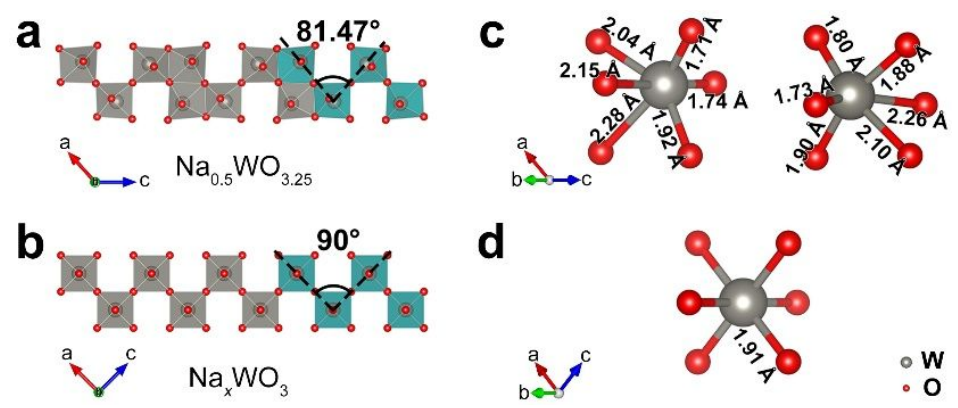

Figure S3. $(\mathrm{a}, \mathrm{b})$ Atomic arrangements of the octahedral chains parallel to $(100)_{\mathrm{T}}$ and $(101)_{\mathrm{C}}$ planes viewed along $[010]_{\mathrm{T}, \mathrm{C}}$, respectively. (c, d) Schematic illustrations of $\mathrm{WO}_{6}$ octahedra in $\mathrm{Na}_{0.5} \mathrm{WO}_{3.25}$ and $\mathrm{Na}_{x} \mathrm{WO}_{3}$, respectively. 

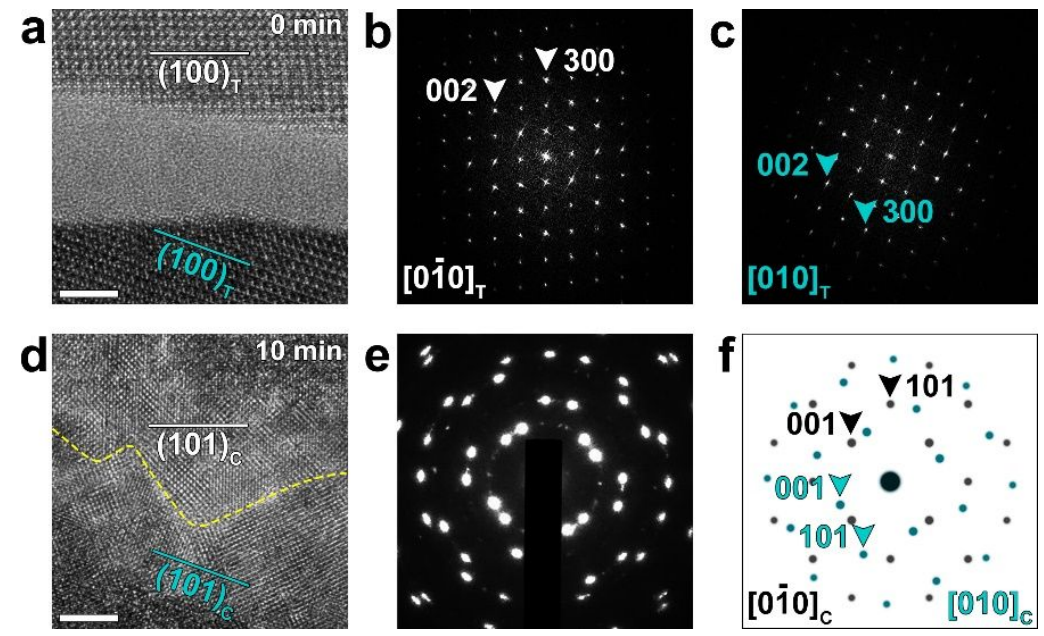

Figure S4. (a) HRTEM image of amorphous between two $\mathrm{Na}_{0.5} \mathrm{WO}_{3.25}$ crystals. (b) and (c) FFTs obtained from the upper and lower crystal regions in panel a, respectively. (d) HRTEM image of the product after e-beam irradiation. The dashed yellow lines depict the boundaries between two domains. (e) Corresponding SAED pattern of panel $d$ and (f) simulated diffraction pattern. Scale bars, $5 \mathrm{~nm}$.
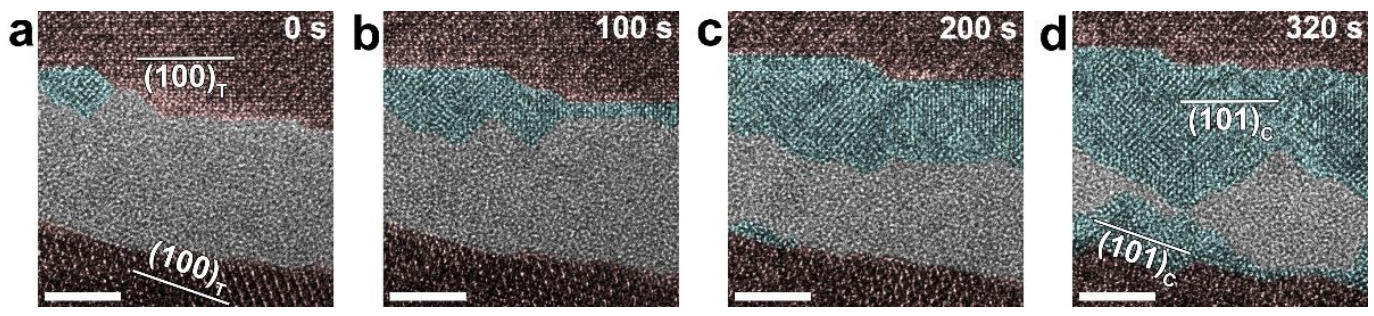

Figure S5. Sequential HRTEM images illustrating the crystallization of the amorphous region. All scale bars, $5 \mathrm{~nm}$.

Movie 1. Atomistic observation of $(100)_{\mathrm{T}} /(101)_{\mathrm{C}}$ phase boundary evolution during the $\mathrm{Na}_{0.5} \mathrm{WO}_{3.25} \rightarrow \mathrm{Na}_{x} \mathrm{WO}_{3}$ phase transition along $[001]_{\mathrm{T}}$ zone axis (play speed $\times 11$ ).

Movie 2. Atomistic observation of $(010)_{\mathrm{T}} /(010)_{\mathrm{C}}$ phase boundary evolution during the 
$\mathrm{Na}_{0.5} \mathrm{WO}_{3.25} \rightarrow \mathrm{Na}_{x} \mathrm{WO}_{3}$ phase transition along $[001]_{\mathrm{T}}$ zone axis (play speed $\times 6.5$ ).

Movie 3. Atomistic observation of $\mathrm{Na}_{0.5} \mathrm{WO}_{3.25} \rightarrow \mathrm{Na}_{x} \mathrm{WO}_{3}$ phase transition projected along $[0 \overline{1} 0]_{\mathrm{T}} \quad$ zone axis (play speed $\times 3$ ).

Movie 4. The crystallization of the amorphous region (play speed $\times 20$ ).

Calculation of temperature rise under e-beam irradiation:

The temperature rise under e-beam irradiation can be calculated by Fisher's model: ${ }^{1}$

$$
\Delta T=\frac{1}{\pi \kappa e}\left(\frac{\Delta E}{d}\right) \ln \underline{b}
$$

where /is the beam current, $k$ is the thermal conductivity of the sample, $e$ is the electron charge $\left(1.602 \times 10^{-19} \mathrm{C}\right), b$ is the sample radius, $r_{0}$ is the radius of electron beam, and $\Delta E$ is the total energy loss of per electron in a sample of thickness $d$. Since $\Delta E$ is ignorable compared with the initial energy $(200 \mathrm{keV}), \Delta E / d$ is equal to the stopping power of electrons $(d E / d x$ ), which can be calculated by the Bethe-Bloch equation:2

$$
-\frac{d E}{d x}=\frac{2 \pi Z \rho\left(e^{2} / 4 \pi \varepsilon\right)^{2}}{m v^{2}}\left\{\begin{array}{c}
\operatorname{In}\left[\frac{E\left(E+m c^{2}\right)^{2} \beta^{2}}{2 r_{e}^{2} m c^{2}}\right]+\left(1-\beta^{2}\right) \\
-\left(2-\sqrt{1-\beta^{2}}-1+\beta^{2}\right) \ln 2 \\
+\frac{1}{8}\left(1-\sqrt{1-\beta^{2}}\right)^{2}
\end{array}\right\}
$$

In this equation, $Z$ is the atomic number of the sample, $\rho$ is the atomic density, $\varepsilon_{0}$ is the dielectric constant $\left(8.85 \times 10^{-12} \mathrm{~F} / \mathrm{m}\right), m$ is the electron rest mass $\left(9.3 \times 10^{-31} \mathrm{~kg}\right), v$ is the 
velocity of the incident electrons, $c$ is the light speed $\left(3 \times 10^{8} \mathrm{~m} / \mathrm{s}\right), E$ is the energy of electron, $l_{\mathrm{e}}$ is the average excitation energy for electrons in the target, and $\beta=v / c$

In this work, the acceleration voltage is $200 \mathrm{kV}$, and thus $v=2.1 \times 10^{8} \mathrm{~m} / \mathrm{s}, \beta=0.7$. For $\mathrm{Na}_{x} \mathrm{WO}_{3}(x=0.5), Z=22.21, \rho=3.77 \times 10^{30} \mathrm{~m}^{-3}, k=15 \mathrm{~W} /(\mathrm{mK}) \cdot{ }^{3}$ With the experimental parameters of $/=2.5 \mathrm{nA}, b=700 \mathrm{~nm}, r_{0}=40 \mathrm{~nm}$, the temperature rise can be estimated to be $2.2 \mathrm{~K}$.

\section{REFERENCES}

(1) Jenčič, I.; Bench, M. W.; Robertson, I. M.; Kirk, M. A. Electron - beam - induced crystallization of isolated amorphous regions in $\mathrm{Si}, \mathrm{Ge}, \mathrm{GaP}$, and $\mathrm{GaAs}$. J. Appl. Phys. $1995,78,974-982$.

(2) Fisher, S. B. On the temperature rise in electron irradiated foils. Radiat. Eff. 1970, 5, 239-243.

(3) Shanks, H. R.; Redin, R. D. The thermal conductivity of some sodium tungsten bronzes. J. Phys. Chem. Solids 1966, 27, 75-78. 